\title{
ATITUDES EM RELAÇÃO À MATEMÁTICA E DESEMPENHO ACADÊMICO: ALGUMAS RELAÇÕES
}

\author{
Renata Sossai Freitas Faria, Maria Alice Veiga Ferreira de Souza, Luiz Henrique Lima \\ Faria
}

Instituto Federal de Educação, Ciência e Tecnologia do Espírito Santo

Vitória, Espírito Santo

E-mail: renatasossai@hotmail.com,alicevfs@hotmail.com,luizlima@ifes.edu.br

\begin{abstract}
Resumo: Esse artigo relata parte de uma investigação maior cujo recorte teve como objetivo investigar as possíveis influências do fator atitude em relação à matemática sobre o desempenho acadêmico nessa disciplina. Para o estudo da atitude, foi aplicada uma escala em uma amostra de 608 alunos pertencentes a uma escola da rede pública municipal de ensino fundamental de Vila Velha-ES. Pelo lado do desempenho em Matemática, foram tomadas as notas desses alunos medidas em provas e trabalhos individuais ou em grupos do tipo lápis e papel desenvolvidas em sala de aula de Matemática ao longo de um semestre letivo. Com essa base de dados, foi possível por meio de instrumentos estatísticos, verificar se a atitude explicava de forma significativa o comportamento da variável dependente desempenho em matemática. Os resultados desta pesquisa revelaram haver influência significativa da variável explanatória sobre a variável dependente ao nível de significância de 0,01.
\end{abstract}

Palavras-chave: matemática, desempenho acadêmico em matemática, atitudes.

\section{ATTITUDES IN RELATION TO MATHEMATICS AND ACADEMIC PERFORMANCE: SOME RELATIONS}

Abstract: This paper reports part of a larger investigation which cut aimed to investigate the possible influences of attitude factor in mathematics on academic performance in this discipline. To study the attitude was applied a scale on a sample of 608 students belonging to a school of public municipal elementary school of Vila Velha-ES. On the performance side in mathematics, notes were taken of these students measured in individual or group tests type pencil and paper developed in mathematics classroom over a semester. With this database, it was possible through statistical tools, verify if the attitude explained significantly the behavior of mathematics performance dependent variable. The results of this research revealed that there is significant influence of the explanatory variable on the dependent variable to the 0.01 significance level.

Keywords: mathematics, academic performance in mathematics, attitudes.

Recebido em 02/04/2015. Publicado em 30/09/2016. 


\section{INTRODUÇÃO}

A Educação Matemática tem buscado auxílio, frequentemente, em teorias da Psicologia Cognitiva para apoiar investigações que expliquem o comportamento de estudantes frente ao estudo da Matemática. Particularmente, interessa-nos aqui investigar a existência de influência da atitude, uma variável psicossocial, sobre o desempenho acadêmico de estudantes na disciplina de Matemática, podendo, assim, contribuir para ampliar a compreensão dos efeitos de variáveis invisíveis, por assim dizer, que silenciosamente, podem explicar os resultados acadêmicos positivos e negativos acerca dessa disciplina.

Nesse sentido e seguindo o mainstream de investigações que buscam fatores que possam explicar a heterogeneidade de desempenho acadêmico na disciplina de matemática (Neves, 2002; Dobarro \& Brito, 2010; Ramírez, 2005; Souza \& Brito, 2008; Machado, 2013), perseguimos responder o seguinte questionamento: a variável psicológica atitude em relação à matemática influencia o desempenho acadêmico dos alunos na disciplina de matemática?

Tendo em vista essa questão de pesquisa, este estudo propõs alcançar o seguinte objetivo (OG) e verificar a seguinte hipótese (HP):

OG - Verificar a relação entre a variável atitude em relação à matemática e o desempenho acadêmico em matemática.

HP - Baseados no fato de que o ser humano está imerso em contextos culturais, sociais, e de outras ordens, e que esses contextos influenciam seu modo de ver entender o mundo em que vive, acreditamos existir alguma relação entre a variável atitude e o desempenho acadêmico em matemática.

\section{REFERENCIAL TEÓRICO}

Tendo como base a revisão de literatura, neste tópico são apresentados, de forma mais específica, teorias e estudos que fundamentaram e justificaram as argumentações no curso deste artigo.

\subsection{Desempenho acadêmico}


No âmbito da Psicologia Educacional, tem sido conferido um enfoque especial ao estudo dos fatores envolvidos no processo de aprendizagem, na medida em que são pensados como executores de um grande papel na motivação e no desempenho acadêmico dos alunos.

No que se refere a esse desempenho, foco de estudo deste trabalho, Souza e Brito (2008) salientam que o desempenho funciona, na prática educacional, como uma ferramenta de medição do aproveitamento educacional pelos alunos no processo de aprendizado de um dado conteúdo, podendo também assinalar para a adequação ou não das diferentes práticas de ensino. Referindose, ainda, à questão do desempenho, Brito $(1996$, p.52) ressalta que:

o desempenho dos indivíduos é avaliado através de vários procedimentos, sendo os mais comuns, as provas e os trabalhos individuais e em grupo. A estas atividades são atribuídas notas e são essas notas que refletem o que é entendido pelo "bom" e "mau" desempenho.

Dessa forma, para o desenvolvimento deste trabalho foi considerado como desempenho acadêmico as notas alcançadas pelos os alunos medidas por provas e trabalhos individuais ou em grupos, do tipo lápis e papel, desenvolvidas em sala de aula na disciplina de matemática dentro de um período de um semestre letivo.

\subsection{Atitude}

O termo atitude, de acordo com Brito (1996), tem sido utilizado com diferentes significados que, dependendo do campo teórico em que se insere, pode ser tratado como sinônimo de crenças, valores, motivação, comportamento, gosto, habilidades, hábito, o que tem gerado controvérsias entre os teóricos.

Particularmente, a teoria sócio-cognitiva, com a qual nos apoiaremos, centra-se no estudo do comportamento humano quando inserido em contexto social dando valor aos processos cognitivos dos indivíduos e aí se encontram as atitudes, ao contextualizar a forma como os fatores psicossociais se relacionam. Nessa mesma direção, Bandura (1986, p.18) pontua que "os fatores pessoais (crenças, expectativas, atitudes e conhecimento), o ambiente (recursos, consequências de ações e ambiente físico), assim como o comportamento (atos e escolhas individuais e declarações verbais), todos influenciam e são influenciados uns pelos outros". 
Além disso, Eagly e Chaiken (1993) esclarecem que as atitudes não podem ser diretamente observáveis, pois estão relacionadas à predisposição que uma pessoa tem para avaliar determinado objeto, seja aprovando-o ou desaprovando-o. Desse modo, as atitudes se reportam à categoria dos construtos, aquilo que é elaborado ou sintetizado com base em dados simples, um conceito, podendo ser inferidas com base em respostas avaliativas que o sujeito emite em relação ao objeto da atitude, tendo como suporte componentes cognitivos, afetivos e conativos.

Já para Brito (1996, p.3), de modo geral, atitude acaba sendo entendida como sinônimo de comportamento, em um enfoque que prioriza apenas o aspecto observável, colocando-a como equivalente à motivação e outros. Para ela, a atitude pode até ser um dos componentes do comportamento, mas não são sinônimos, e não podem ser confundidos.

Ainda segundo essa autora, a análise da literatura a respeito das atitudes com relação à matemática mostra que os termos atitudes, crenças e valores são, muitas vezes, empregados como sinônimos, o que gera confusão e obscurece ainda mais a atribuição de significado.

Assim, a ideia de atitude adotada na presente pesquisa é a conceituada por Brito $(1996, p .11)$ que nos ensina: "Atitude é definida como uma disposição pessoal, idiossincrática, presente em todos os indivíduos, dirigida a objetos, eventos ou pessoas, que assume diferente direção e intensidade de acordo com as experiências do indivíduo. Além disso, apresenta componentes de domínio afetivo, cognitivo e motor.".

Fundamentados nos estudos apresentados neste referencial teórico, além de Jesus (2005), Soares (2003), Fuentes, Lima \& Guerra (2009), Faria (2006) e Aiken (1970), seguiremos discorrendo sobre o método utilizado para alcance dos fins que desejamos.

\section{MÉTODO, PARTICIPANTES, INSTRUMENTOS E PROCEDIMENTOS}

O presente trabalho, de caráter quantitativo, caracteriza-se como ex-post-facto do tipo correlacional, que se assemelha, de acordo com Gil (1999) à pesquisa experimental, uma vez que se constitui em uma investigação sistemática e empírica, não tendo controle direto sobre as variáveis independentes porque já ocorreram suas manifestações. O método está baseado em inferências sobre a relação entre variáveis sem observação direta, a partir de indicadores de variação concomitante entre as variáveis independentes e dependentes. 
Participaram da investigação 608 estudantes de uma escola do município de Vila Velha-ES que atende estudantes do Ensino Fundamental em idade regular - do 1으 ao 9o anos - e na modalidade de educação de jovens e adultos (EJA) - 1a a 8a séries. Desses 608,498 cursavam do 3ำ ao 9o anos, distribuídos quase que igualitariamente na amostra segundo o ano que cursavam e, os outros 110 faziam parte da EJA distribuídos nas oito séries.

Para o estudo das atitudes, foi utilizada uma escala de atitudes em relação à matemática como instrumento de pesquisa. A escala de atitudes em relação à matemática é uma escala do tipo Likert e consta de vinte afirmações que tentam expressar o sentimento que cada estudante possui em relação à matemática, sendo dez afirmações positivas (afirmações 03, 04, 05, 09, 11, 15, 16, 19,20 ) e dez afirmações negativas (afirmações 01, 02, 06, 07, 08, 09, 10, 12, 13 e 17).

Os participantes escolheram, para cada afirmação, uma das quatro alternativas: discordo totalmente, discordo, concordo, concordo totalmente. Para cada item escolhido foi atribuído um número de pontos de 1 a 4. Para afirmações positivas, a ordem de atribuição dos valores foi 1, 2, 3 e 4. Para afirmações negativas, a ordem foi inversa, ou seja, 4, 3, 2 e 1 . O somatório de valores desse instrumento varia de 20 a 80 pontos possíveis, indicando, respectivamente, atitudes mais positivas e mais negativas. Para o alcance dos objetivos deste trabalho, não foram necessárias análises dos escores de cada fator, mas sim o escore total da escala. A título de exemplificação, a figura 1 apresenta um estrato da escala utilizada.

Figura 1. Estrato da escala de atitudes em relação à matemática.

Instruções: Cada uma das frases a seguir expressa o sentimento que cada pessoa apresenta com relação à Matemática. Você deve comparar o seu sentimento pessoal com aquele expresso em cada frase, assinalando um dentre os quatro pontos colocados abaixo de cada uma delas, de modo a indicar com a maior exatidão possível, o sentimento que você experimenta com relação à Matemática.

01. Eu fico sempre sob uma terrível tensão na aula de Matemática.

( ) Discordo Totalmente () Discordo ( ) Concordo () Concordo Totalmente

02. Eu não gosto de Matemática e me assusta ter que fazer essa matéria.

( ) Discordo Totalmente ( ) Discordo ( ) Concordo ( ) Concordo Totalmente

Fonte: Brito (1996; 1998). 
No que concerne ao desempenho acadêmico, para os fins deste trabalho, foram colhidas as médias trimestrais dos alunos na disciplina de matemática, disponibilizadas por meio de uma listagem fornecida pelo sistema acadêmico da escola.

Com os escores totais da escala e das médias escolares semestral dos participantes na disciplina de matemática, foi possível realizar as análises estatísticas necessárias para o alcance dos objetivos. Esses procedimentos estatísticos (correlações, regressões e testes) foram elaborados com auxílio dos softwares estatísticos MINITAB 16 e SPSS 22.

O tratamento dos dados foi subdividido em duas etapas que passamos a apresentar:

Etapa 1 - Verificou-se o grau de associação entre a variável atitude e o desempenho acadêmico que varia de $-1,00$ a +1,00 para o instrumento estatístico denominado correlação de Pearson. Esse grau pode ser avaliado conforme indicações de Hair et all (2010) e que reproduzimos na Tabela 1:

Tabela 1. Força de associação entre as variáveis autoconceito e desempenho acadêmico.

\begin{tabular}{c|c}
\hline Variação do coeficiente & Força da associação \\
\hline $\pm 0,91$ a $\pm 1,00$ & Muito forte \\
\hline $\pm 0,71$ a $\pm 0,90$ & Alta \\
\hline $\pm 0,41$ a $\pm 0,70$ & Moderada \\
\hline $\pm 0,21$ a $\pm 0,40$ & Pequena mas definida \\
\hline $\pm 0,01$ a $\pm 0,20$ & Leve, quase imperceptível \\
\hline \multicolumn{2}{r}{ Fonte: Hair et al. (2010). }
\end{tabular}

Etapa 2 - Verificou-se por meio do instrumento estatístico regressão linear se a variável independente atitude explicava o comportamento da variável dependente desempenho acadêmico em matemática. Essa verificação foi precedida pelo teste de normalidade de RyanJoiner, que é um pressuposto para o uso da regressão linear, sendo, por fim, atestada a simetria dos dados. Ademais, após a realização da regressão, os pressupostos de normalidade dos resíduos e homocedasticidade foram examinados, respectivamente, pelos métodos Teste de Normalidade Ryan-Joiner e Teste de Pesaran- Pesaran.

De acordo com Dancey e Reidy (2013), a análise de regressão é uma extensão da análise de correlação. Enquanto a análise de correlação permite determinar a força da relação entre duas variáveis (tanto magnitude quanto direção), a regressão linear informa quanto do comportamento 
de uma variável dependente é afetado pelo comportamento da variável independente, segundo a expressão (1) seguinte:

$$
D A_{i}=\alpha+\mu \cdot A t_{i}+e_{i}
$$

segundo a qual, foi verificado o efeito da variável explanatória atitudes em relação à matemática em confronto com a variável dependente desempenho acadêmico em matemática. Nessa expressão, DAi representa o desempenho acadêmico por estudante previsto pela regressão; ? é a média dos desempenhos acadêmicos; $\mu$ é o coeficiente angular da variável independente atitudes em relação à matemática por estudante que é representada por Ati; por fim, ei representa o resíduo provocado por outras variáveis independentes ausentes do modelo. Após a utilização do modelo, por meio do $\mathrm{R}^{2}$ e do teste $\mathrm{F}$, é possível aceitar ou rejeitar a hipótese HP.

A investigação contou com a autorização dos participantes, registrada e assinada em Termo de Consentimento Livre e Esclarecido, no qual constam, entre outros compromissos que envolvem a pesquisa, o de preservação da identidade dos participantes em todos os instrumentos de aplicação para a coleta de dados.

\section{RESULTADOS E ANÁLISE DE DADOS}

Nesta seção são apresentados os resultados do tratamento estatístico ao qual os dados foram submetidos, seguidos das respectivas análises.

A tabela 2 expõe a associação entre a variável atitude e a variável desempenho acadêmico pela correlação de Pearson $(0,462)$, ao nível de 0,01. Esse resultado demonstra existir moderada dependência entre essas variáveis, levando, portanto, a aceitar HP.

Tabela 2. Análise correlação para associação das variáveis de Autoconceito e Desempenho Acadêmico.

\begin{tabular}{ll|c|c}
\hline & Desempenho Acadêmico & Atitudes \\
\hline Desempenho Acadêmico & Correlação de Pearson & 1 & 0,462 \\
& Sig. (2 extremidades) & & 0,000 \\
& $\mathrm{~N}$ & 608 & 608 \\
\hline Atitudes & Correlação de Pearson & 0,462 & 1 \\
& Sig. (2 extremidades) & 0,000 & 608 \\
& $\mathrm{~N}$ & 608 & 6
\end{tabular}


** A correlação é significativa no nível 0,01.

Fonte: dados tratados pela primeira autora no software MINITAB $23{ }^{\circledR}$.

A análise da relação entre a variável independente atitude em relação à matemática e a variável dependente desempenho acadêmico em matemática, por meio de regressão linear, obteve o seguinte resultado expresso na Tabela 3:

Tabela 3. Análise da relação entre a variável independente atitude em relação à matemática e a variável dependente

\begin{tabular}{c|c|c|c|c|c}
\multicolumn{7}{|c|}{} & $\begin{array}{c}\text { Graus de } \\
\text { Liberdade }\end{array}$ & $\begin{array}{c}\text { Soma dos } \\
\text { Quadrados }\end{array}$ & Média da Soma & F & Significância \\
\hline Regressão & 1 & 3,2058 & 3,2058 & 164,76 & 0,000 \\
\hline Resíduo & 606 & 11,7911 & 0,0195 & & \\
\hline Total & 607 & 14,9969 & & & \\
\hline $\mathbf{R}^{2}$ & 0,214 & & & &
\end{tabular}

Fonte: Dados tratados pela primeira autora no software MINITAB $23{ }^{\circledR}$.

O fator atitudes em relação à matemática explica $\left(R^{2}=0,214\right)$ o comportamento da variável dependente desempenho em matemática em $21,4 \%$ dos casos. $O$ teste $F$, por sua vez, apresentou o resultado de 164,76, revelando, com significância de 0,000, que o modelo de regressão linear simples é apropriado, determinando a existência de relação significativa entre a variável dependente e a variável explanatória. O resultado do teste de normalidade Ryan-Joiner, bem como o resultado do teste de homocedasticidade de Pesaran-Pesaran aplicado aos resíduos da regressão, confirmou o cumprimento dos pressupostos da regressão, e, com isso, pôde-se afirmar a influência do fator atitude em relação à matemática sobre o desempenho acadêmico nessa disciplina.

\section{CONSIDERAÇÕES FINAIS}

Os resultados deste estudo apresentaram contribuições que se prestam a conhecer relações existentes entre aspectos cognitivos - no nosso caso as atitudes - e os aspectos relacionados à educação matemática (desempenho acadêmico em matemática).

As relações entre os aspectos psicossociais e o desempenho em matemática, apesar de já existirem em outras investigações, ainda carecem de uma visão mais ampla sobre o fenômeno, inclusive com estudantes diferenciados, necessitando, assim, que mais estudos contribuam para o conhecimento dessas intrincadas relações. 
Neste trabalho, constatou-se relação entre as atitudes dos estudantes em relação à matemática e o seu desempenho acadêmico nessa disciplina. Esse resultado confirma as conclusões de outros estudos como os de Araújo (1999) e Brito (1996), que revelaram que os alunos que apresentaram maiores valores na escala de atitudes em relação à matemática, apresentavam maior desempenho acadêmico nessa disciplina. Aqui, as atitudes em relação à matemática possuem uma influência de $21 \%$ sobre o desempenho acadêmico em matemática. Isto significa que, quanto mais positiva for a atitude do aluno em relação à matemática, maior será seu o desempenho acadêmico nessa disciplina, uma vez que a correlação foi positiva. Esse resultado deixa a mensagem de que esforços educacionais que gerem atitudes positivas em relação à matemática em seus estudantes refletirse-ão, provavelmente, sobre os seus desempenhos escolares nessa disciplina.

Cabe mencionar que o presente estudo encontrou evidências que não foram mais profundamente exploradas, por fugir de seu escopo, de que o fator ano/série pode alternativamente ser utilizado como variável moderadora nas relações entre as atitudes em relação à matemática e o desempenho acadêmico em matemática. Sugere-se, portanto, continuidade da investigação em futuras pesquisas.

\section{REFERÊNCIAS}

AIKEN, L. R. Attitudes Toward Mathematics. Review of Educational Research. v. 40, n. 4, p. 551-596, 1970.

ARAÚJO, E. A. Influência das habilidades e das atitudes em relação à matemática e à escolha profissional. 228 p. Teses (Doutorado). Faculdade de Educação, Universidade Estadual de Campinas, Campinas, 1999.

BANDURA, A. Social foundations of thought and action: a social cognitive theory. New Jersey: Prentice Hall, 1986.

BRITO, M. R. F. Um estudo sobre as atitudes em relação à matemática em estudantes de 10 e 20 graus. Campinas, SP: Faculdade de Educação da UNICAMP, 1996.

Adaptação e validação de uma escala de atitudes em relação à Matemática. Zetetiké, Campinas, v. 6, n. 9, p. 109-162, 1998.

DANCEY, C. P.; REIDY, J. Estatística sem matemática para psicologia. Tradução técnica: Lori Viali. 5.ed. Porto Alegre: Penso, 2013.

DOBARRO, V. R.; BRITO, R. F. Atitude e crença de autoeficácia: relações com o desempenho em matemática. Educ. Matem. Pesq., São Paulo, v.12, n.2, p.199-220, 2010.

EAGLY, A. H., \& CHAIKEN, S. The psychology of attitudes. Belmont, California: Wadsworth Group/Thomson Learning, 1993.

FARIA, P. C. Atitudes em relação à matemática de professores e futuros professores. 2006. 332 f. Tese (Doutorado em Educação). Faculdade de Educação, Universidade Estadual de Campinas, Campinas, 2006. 
FUENTES, V. L. P.; LIMA, R.; GUERRA, D. D. S. Atitudes em relação à matemática em estudantes de Administração. Revista Semestral da Associação Brasileira de Psicologia Escolar e Educacional (ABRAPEE), v.13, n.1, Janeiro/Junho de 2009. 133-141. Disponível em: <http://www.scielo.br/pdf/pee/v13n1/v13n1a15.pdf>. Acesso em: 22 nov. 2012.

GIL, A. C. Métodos e Técnicas de Pesquisa Social. Editora: Atlas, 1999.

HAIR, J. R. J. F; BABIN, B.; MONEY, A. H.; SAMOUEL, P. Fundamentos de métodos de pesquisa em administração. Porto Alegre: Bookman, 2010.

JESUS, M. A. S. de. As atitudes e o desempenho em operações aritméticas do ponto de vista da aprendizagem significativa. Campinas, SP: [s.n.], 2005.

MACHADO, M. C. Um estudo sobre as atitudes em relação à matemática, crenças de autoeficácia matemática e o desempenho acadêmico dos estudantes. Curitiba: Anais do XI Encontro Nacional de Educação Matemática, 2013.

NEVES, L. F. Um estudo sobre as relações entre a percepção e as expectativas dos professores e dos alunos e o desempenho em Matemática. Campinas, SP: 2002.

RAMÍREZ, M. J. Attitudes toward mathematics and academic performance among chilean 8th graders. Estudios Pedagógicos XXXI, n.1, p. 97-112, 2005.

SOARES, F. G. E. P. As atitudes de alunos do ensino básico em relação à matemática e o papel do professor. Campo Grande: Universidade Católica Dom Bosco, 2003.

SOUZA, L. F. N. I. D.; BRITO, M. R. F. D. Crenças de auto-eficácia, autoconceito e desempenho em matemática. Estud. psicol. vol.25, n.2, p. 193-201. Campinas, 2008. 